\title{
A Case of Acute Bilateral Retrocochlear Hearing Loss as an Initial Symptom of Unilateral Thalamic Hemorrhage
}

\author{
Min-Joon Park, Sung-Won Yoon, Kang-Hyeon Kim, and Young-Jin Kim \\ Department of Otorhinolaryngology-Head and Neck Surgery, Bundang Jesaeng General Hospital, Daejin Medical Center, \\ Seongnam, Korea
}

$\begin{array}{ll}\text { Received } & \text { February 26, 2014 } \\ \text { Revised } & \text { April 29, 2014 } \\ \text { Accepted } & \text { May 10, 2014 }\end{array}$

Address for correspondence

Young-Jin Kim, MD

Department of Otorhinolaryngology-

Head and Neck Surgery,

Bundang Jesaeng General Hospital,

Daejin Medical Center,

20 Seohyeon-ro 180beon-gil,

Bundang-gu, Seongnam 463-774,

Korea

Tel +82-31-779-0260

Fax $+82-31-779-0265$

E-mail yj@dmc.or.kr
A speech discrimination test is a test using a list of 25 phonetically balanced monosyllables. It is often overlooked but significant enough for pure tone audiometry. Many physicians have performed pure tone audiometry but without a speech discrimination test. A 73-year-old woman visited our clinic complaining of sudden bilateral hearing loss. Pure tone audiometry showed only bilateral high frequency loss. However, speech discrimination had decreased markedly. We decided to follow-up after 1 week of Ginexin- $\mathrm{F}^{\circledR}$ (ginkgo leaf extract) and Nafril ${ }^{\circledR}$ (nafronyl oxalate). She felt a gait disturbance within 2 days. Magnetic resonance imaging revealed a left thalamic hemorrhage. After a 1 month hospitalization, the hematoma subsided, and speech discrimination recovered 3 months later. Acute hearing loss due to thalamic hemorrhage that recovered has never been reported. We report the first case of retrocochlear hearing loss that occurred with a thalamic hemorrhage in a patient who recovered.

Korean J Audiol 2014;18(2):80-84

KEY WORDS: Thalamus · Retrocochlear disease · Hearing loss · Speech discrimination tests.

\section{Introduction}

Sudden sensorineural hearing loss is defined as a hearing reduction $>30 \mathrm{~dB}$, over at least three contiguous frequencies and occurring within $72 \mathrm{hr} .{ }^{1)}$ The speech discrimination score of a patient suffering from hearing loss may not be considered relative to pure tone audiometry and the hearing test is often only done using pure tone audiometry. However, the results of a speech discrimination test are as important as those of pure tone audiometry for a patient with retrocochlear hearing loss. $^{2)}$

It is difficult to doubt intracerebral hemorrhage or infarction when a patient complains of decreased speech discrimination with no neurologic abnormality. Retrocochlear hearing loss is not a common disease and an acute hearing loss is rare. We often see patients who have slowly progressing hearing loss with poor speech discrimination. An acute thalamic hemorrhage with reversible hearing loss has not reported by

This is an Open Access article distributed under the terms of the Creative Commons Attribution Non-Commercial License (http://creativecommons. org/licenses/by-nc/3.0/) which permits unrestricted non-commercial use, distribution, and reproduction in any medium, provided the original work is properly cited. an otolaryngologist. We report a case of acute bilateral speech discrimination loss as an initial symptom of unilateral thalamic hemorrhage, and the patient recovered.

\section{Case Report}

A 73-year-old woman visited our clinic complaining of acute bilateral hearing loss that occurred $7 \mathrm{hr}$ previously. The patient showed no neurological deficits. Otoscopy showed bilateral normal tympanic membranes. A pure tone audiometry test revealed bilateral high frequency loss, and pure tone averages were $25 \mathrm{~dB}$ in each side. However, speech discrimination scores were $44 \%$ on the right and $56 \%$ on the left (Fig. 1). We decided to follow-up audiometry after 1 week of Ginexin- $\mathrm{F}^{\mathbb{B}}$ (ginkgo leaf extract, SK Chemical Co., Seoul, Korea) and $\mathrm{Nafril}^{\circledR}$ (nafronyl oxalate, Hanall Biopharma, Seoul, Korea) treatment.

She returned with a gait disturbance after 2 days. Magnetic resonance imaging (MRI)(Fig. 2) and computed tomographyangiography (Fig. 3) revealed a left thalamic hemorrhage. The patient was hospitalized and treated with $20 \%$ mannitol injection for managing high blood pressure and was put on ab- 
Fig. 1. Initial audiogram. Pure tone audiometry was normal; speech discrimination had decreased.
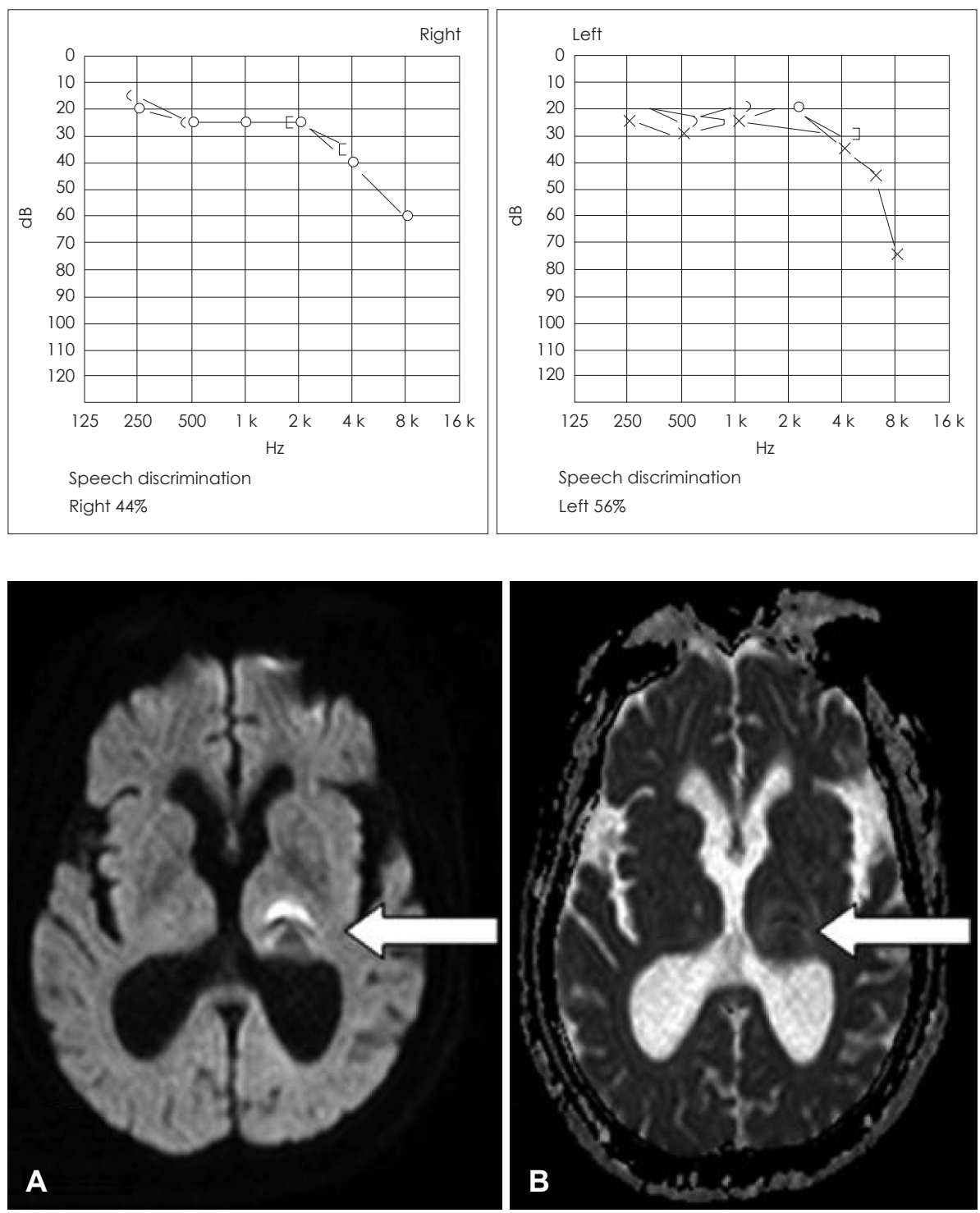

Fig. 2. Diffusion-weighted magnetic resonance image. A: Diffusion image. B: Apparent diffusion coefficient map. Each arrow shows restricted diffusion in the left thalamus.

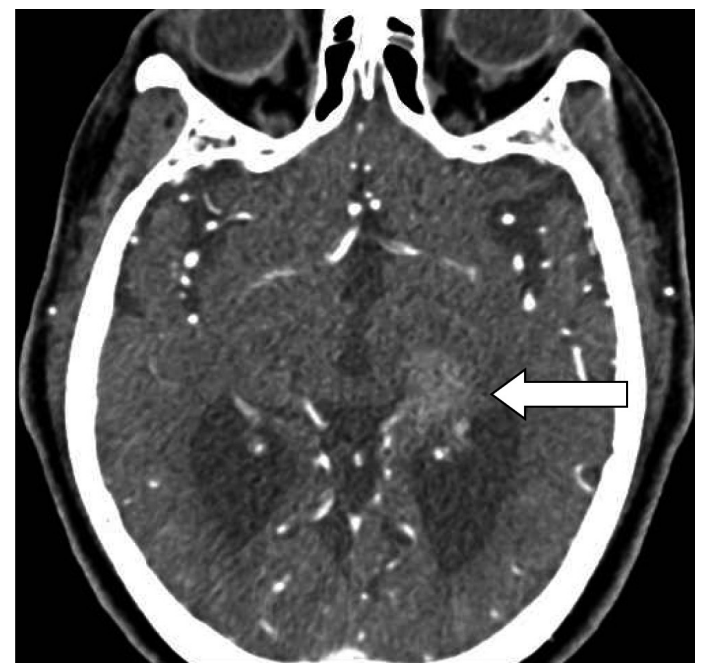

Fig. 3. Brain computed tomography angiography. Enhanced image. Arrow indicates high attenuation in the left thalamus. solute bed rest. She developed dysarthria and right hemiparesis. On hospital day 20, the pure tone audiometry scores were the same, and speech discrimination scores were further reduced to $28 \%$ on the right and $44 \%$ on the left (Fig. 4). The auditory steady state response threshold was $80 \mathrm{~dB}$ on the right and $87 \mathrm{~dB}$ on the left (Fig. 5). She recovered from dysarthria and right hemiparesis on hospital day 21 and went home. Audiometry was rechecked 5 months later. She felt subjective hearing improvement. Speech discrimination scores improved on both sides to $84 \%$ (Fig. 6), and the auditory steady-state response threshold improved about 20\% (Fig. 7).

\section{Discussion}

Thalamic hemorrhage constitutes $6-25 \%$ of intracerebral hemorrhages. ${ }^{3)}$ Fisher described three clinical signs to consid- 
er for a thalamic hemorrhage: 1) predominance of sensory deficits over motor deficits; 2) oculomotor signs including abnor- malities in vertical gaze; and 3) language disturbances due to dominant-hemisphere thalamic hematomas. Consciousness

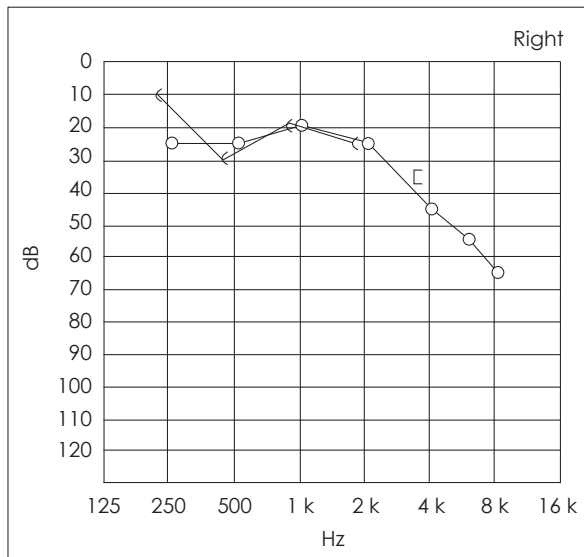

Speech discrimination Right $28 \%$

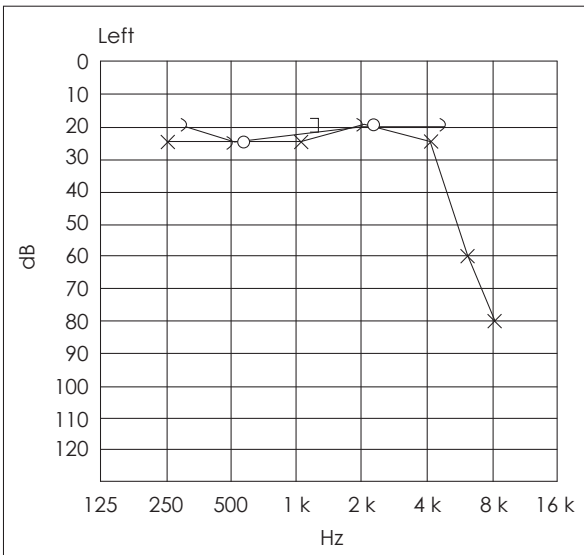

Speech discrimination Left $44 \%$
Fig. 4. Audiogram (3 weeks later). Pure tone audiometry was normal, whereas speech discrimination had decreased.

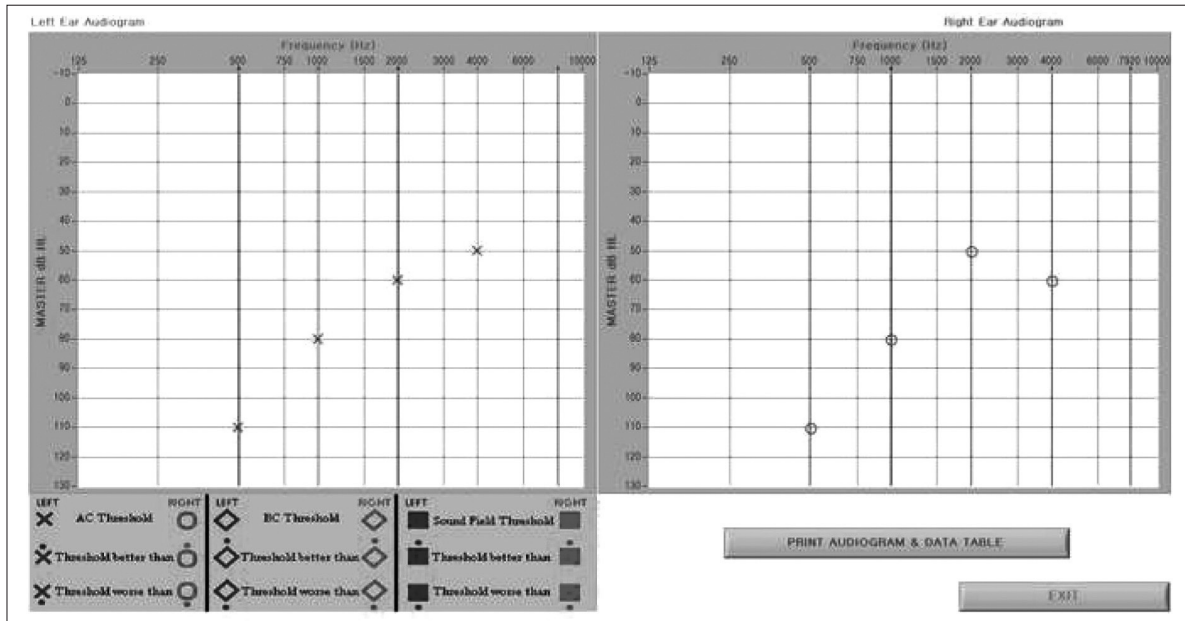
Lt $\quad 500 \quad 1 k \quad 2 k \quad 4 k$
Rt $500 \quad 1 k \quad 2 k \quad 4 k$
$110 \quad 80 \quad 60 \quad 50$
$110 \quad 80 \quad 50 \quad 60$

Fig. 5. Initial auditory steady-state response. Thresholds were lower than pure tone audiometry at all frequencies.

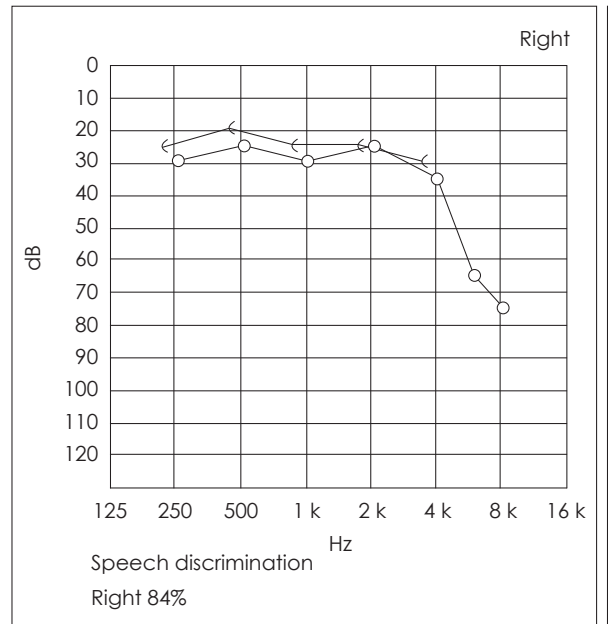

Fig. 6. Audiogram (5 months later). Pure tone audiometry was normal and speech discrimination had improved. 
Fig. 7. Follow up auditory steadystate response (5 months later). Thresholds improved from 0.5-1 $\mathrm{kHz}$.

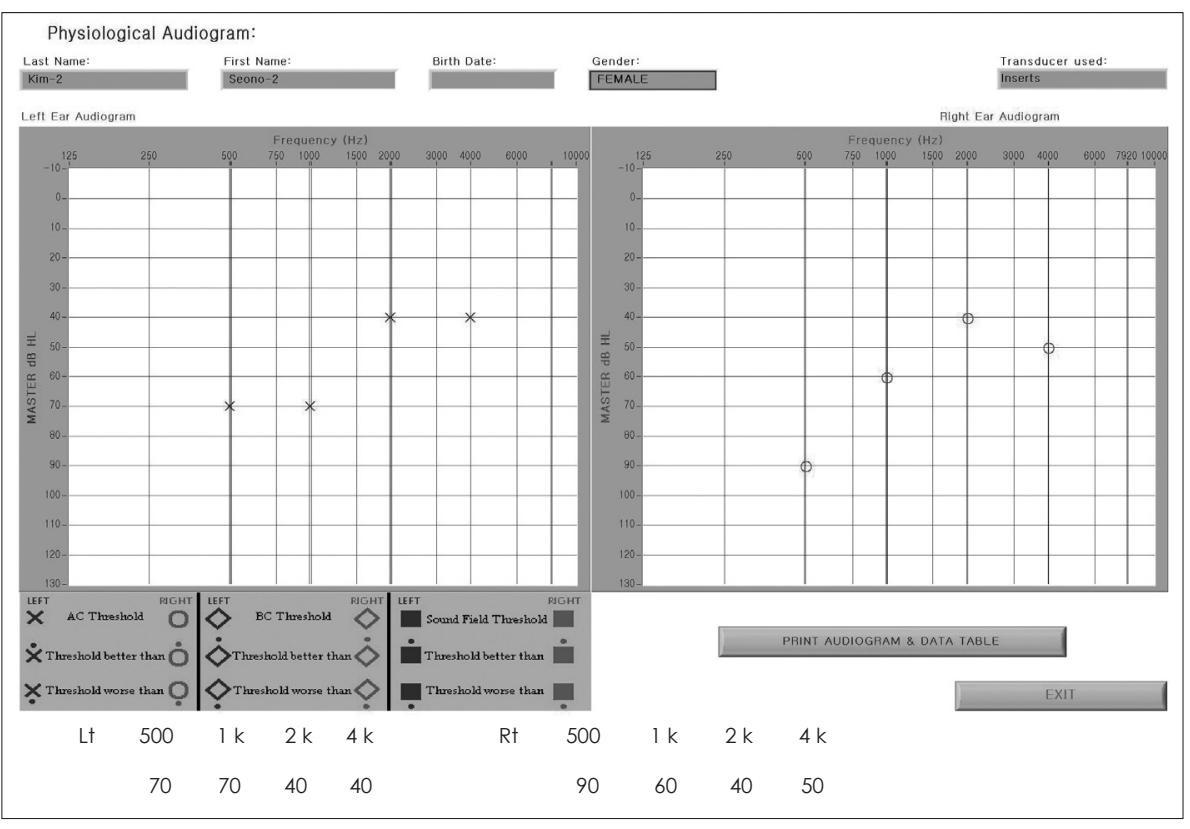

level at the beginning is a predictor of mortality. ${ }^{4)}$ Our patient was old and had hypertension, but the sudden hearing decrease was the only symptom, as no neurologic abnormality was detected. Thus, we did not doubt an intracranial hemorrhage.

In this case, pure tone audiometry from $500 \mathrm{~Hz}$ to $4 \mathrm{kHz}$ was normal but the bilateral speech discrimination scores were very low. These results are characteristic of retrocochlear hearing loss. She showed neurologic abnormalities 2 days later and was diagnosed with a left thalamic hemorrhage on an imaging study.

The central auditory pathway consists of the cochlear nucleus, superior olivary complex, inferior colliculus, medial geniculate body, and auditory cortex. ${ }^{5)}$ The medial geniculate body (MGB) is the main auditory-responsive portion in thalamus, which consists of ventral, dorsal, and medial subdivisions. The MGV (the ventral subdivision of the MGB) is the core subdivision and rapidly transmits sharp tone auditory information to the auditory cortex. The MGD (the dorsal division of the MGB) is a slower, more integrative subdivision and is influenced strongly by non-auditory inputs, such as visual or associating sounds. The MGM (the medial division of MGB) includes diverse regions such as the suprageniculate nucleus, the posterior intralaminal nucleus, and the peripedencular nucleus. An abnormality in this pathway leads to retrocochlear hearing loss. ${ }^{6}$

The patient's general condition had improved 3 weeks later, but the follow-up speech test results were worse, and auditory steady state thresholds were high and different from the pure tone audiometry.

Why did bilateral speech discrimination scores decrease with a unilateral thalamic hemorrhage? Many studies have reported unilateral hearing loss due to unilateral retrocochlear pathology of varying degrees. ${ }^{7)}$ Calford ${ }^{8)}$ reported that $60 \%$ of MGB neurons can be excited by stimuli to either ear, but $20-30 \%$ can be excited only by contralateral ear stimulation. About $10-20 \%$ are inhibited by ipsilateral stimulation. Thus, we speculate that a rapid progressive hemorrhage caused deterioration of the synchronous neural responses and decreased sound location tuning. ${ }^{7)}$ Her subjective hearing recovered after 5 months with resolution of the brain hematoma. Thus, it was a reversible hearing loss.

Auditory steady-state response (ASSR) stimulates the auditory brainstem structures at a modulation frequency of $80 \mathrm{~Hz}$. Kawase, et al. ${ }^{7)}$ reported interesting retrocochlear hearing loss cases with ASSR. Four patients with a vestibular schwannoma with absent or only wave I positive auditory brainstem response responses showed poorer speech discrimination, very small $40 \mathrm{~Hz}$ ASSR responses, and a positive N1m response. Their pure tone averages were $15-55 \mathrm{~dB}$. Although our ASSR had a modulation frequency of $80 \mathrm{~Hz}$, it did not represent cortical activity but ABR or ASSR can decrease with better pure tone responses in a patient with a retrocochlear lesion. We supposed it was the influence of dyssynchrony by a neural conduction problem, as progressive hearing loss may be due to the demyelinization of the auditory nerve that recovered. ${ }^{7,9)}$

The interesting finding in this case was that after improvement of the thalamic hemorrhage, speech discrimination and the ASSR average recovered in 5 months.

MRI has been recognized as the most exact tool to determine hearing-impaired or retrocochlear hearing loss due to intrace- 
rebral diseases. However, it is not easy to apply MRI to a patient with an initial diagnosis of hearing loss without neurological symptoms. Furthermore, it seems easy to overlook the possibility of retrocochlear lesion in the initial diagnosis for a patient with hearing loss. Sudden hearing loss can be diagnosed only by pure tone audiometry; therefore, a speech discrimination test should not be avoided for patients suffering from hearing loss.

In conclusion, a speech discrimination test should always be accompanied by pure tone audiometry when hearing testing is conducted. This test provides additional information about the hearing state and helps to detect retrocochlear hearing loss. We report a case of retrocochlear hearing loss due to acute thalamic hemorrhage in a patient who recovered.

\section{REFERENCES}

1) Wilson WR. Why treat sudden hearing loss. Am J Otol 1984;5:481-3.
2) Kim H, Lee YS, Chung JW, Yoon TH, Lee KS. Efficacy of audiologic tests in the differential diagnosis of cochlear and retrocochlear hearing loss. Korean J Otolaryngol-Head Neck Surg 1999;42:955-60.

3) Tokgoz S, Demirkaya S, Bek S, Kasıkcı T, Odabasi Z, Genc G, et al. Clinical properties of regional thalamic hemorrhages. J Stroke Cerebrovasc Dis 2013;22:1006-12.

4) Kwak R, Kadoya S, Suzuki T. Factors affecting the prognosis in thalamic hemorrhage. Stroke 1983;14:493-500.

5) Häusler R, Levine RA. Auditory dysfunction in stroke. Acta Otolaryngol 2000;120:689-703.

6) Bartlett EL. The organization and physiology of the auditory thalamus and its role in processing acoustic features important for speech perception. Brain Lang 2013;126:29-48.

7) Kawase T, Kanno A, Takata Y, Nakasato N, Kawashima R, Kobayashi T. Positive auditory cortical responses in patients with absent brainstem response. Clin Neurophysiol 2014;125:148-53.

8) Calford MB. The parcellation of the medial geniculate body of the cat defined by the auditory response properties of single units. J Neurosci 1983;3:2350-64.

9) Fischer C, Bognar L, Turjman F, Lapras C. Auditory evoked potentials in a patient with a unilateral lesion of the inferior colliculus and medial geniculate body. Electroencephalogr Clin Neurophysiol 1995; 96:261-7. 